\section{AB0988 BONE QUALITY EVALUATION USING THE NEW TRABECULAR BONE SCORE (TBS) TOOL IN RHEUMATOID ARTHRITIS PATIENTS SUPPLEMENTED WITH VITAMIN D}

A. Casabella ${ }^{1}$, S. Paolino ${ }^{1}$, B. Ruaro ${ }^{1}$, A. Sulli ${ }^{1}$, C. Pizzorni ${ }^{1}$, E. Alessandri ${ }^{1}$, D. Fasciolo ${ }^{1}$, C. Seriolo ${ }^{2}$, L. Molfetta ${ }^{2}$, P. Odetti ${ }^{2}$, B. Seriolo ${ }^{2}$, M. Cutolo ${ }^{1}$ ${ }^{1}$ Department of Internal Medicine, Di.M. I. Polyclinic Hospital, San Martino, University of Genoa, Genoa Italy, Research Laboratory and Academic Division of Clinical Rheumatology, ${ }^{2}$ Department of Internal Medicine, Di.M.I. Polyclinic Hospital, San Martino, University of Genoa, Genoa Italy, Osteoporosis, Bone and Joint Disease Research Center, CROPO, Genova, Italy

Background: Patients affected by Rheumatoid Arthritis (RA) show an increased risk of low bone mass, as a result of multi-systemic disorders including toxic drug, low vitamin D levels, use of glucocorticoids and physical inactivity. Trabecular Bone Score (TBS), is an index extracted from the dual-energy X-ray absorptiometry (DXA) images, that provides an indirect measurement (Score) of bone axial microarchitecture and allows to get information about bone quality. ${ }^{1,2}$

Objectives: The aim of this investigation was to evaluate by TBS the bone quality in AR patients (high risk population) receiving vitamin $D$ supplementation from at least 3 months ( $1000 \mathrm{IU} / \mathrm{die})$.

Methods: 108 female patients (mean age $61 \pm 8$ years) affected by RA and 60 age- matched controls (CNT) (mean age 64 \pm 11 years) were analysed in winter time. Bone Mineral Density (BMD, $\mathrm{g} / \mathrm{cm}^{2}$ ) of the lumbar spine (L1-L4) was analysed using a DXA scan (GE, Lunar Prodigy). Lumbar spine TBS (TBS iNsight Medimaps) was derived for each spine DXA examination. All patients were evaluated for serum 25 hydroxyvitamin $\mathrm{D}(25(\mathrm{OH}) \mathrm{D})$ serum concentrations.

Results: RA patients showed lower $25(\mathrm{OH}) \mathrm{D}$ concentrations $(18.4 \pm 1.3 \mathrm{ng} / \mathrm{ml})$ than CNT $(26.2 \pm 0.9 \mathrm{ng} / \mathrm{ml} ; p<0.04)$ possibly due to low dosage and short treatment. Seventy-eight RA patients ( $80 \%$ of study population) presented a bone loss that was significant when compared to the control group $(p<0.001)$. In particular, BMD was found significantly lower in RA patients compared with matched control group (respectively, Lumbar spine: $0.862 \pm 0.194 \mathrm{~g} / \mathrm{cm}^{2}$ vs $1.240 \pm 0.932 \mathrm{~g} / \mathrm{cm}^{2}$; Femoral neck: $0.668 \pm 0.141 \mathrm{~g} / \mathrm{cm}^{2}$ vs $0.845 \pm 0.164 \mathrm{~g} / \mathrm{cm}^{2}$; Ward: $0.486 \pm 0.221 \mathrm{~g} /$ $\mathrm{cm}^{2}$ vs $0.657 \pm 0.106 \mathrm{~g} / \mathrm{cm}^{2}$; Trochanter: $0.598 \pm 0.231 \mathrm{~g} / \mathrm{cm}^{2}$ vs $0.725 \pm 0.143 \mathrm{~g} /$ $\mathrm{cm}^{2}$; Total hip: $0.764 \pm 0.244 \mathrm{~g} / \mathrm{cm}^{2}$ vs $1.033 \pm 0.161 \mathrm{~g} / \mathrm{cm}^{2}$, all $\left.\mathrm{p}<0.001\right)$. Likewise, lumbar spine TBS score was found significantly lower in RA patients when compared with CNT $(0.904 \pm 0.148$ vs $1.361 \pm 0.126$, both $p<0.001)$

Conclusions: This study shows in RA patients a reduction of TBS values that seem placed side by side with reduced BMD values and in presence of serum 25 $(\mathrm{OH}) \mathrm{D}$ insufficiency. A more carefull analysis of the clinical status/treatments should let to better identify RA patients at higher risk of bone loss.

\section{REFERENCE:}

[1]

Cutolo M et al. Autoimmun Rev 2011:12;84-7,2. Avouac J, et al. Arthritis Care Res 2012;64:1871-8

Disclosure of Interest: None declared DOI: 10.1136/annrheumdis-2018-eular.5574

\section{AB0989 THE IMPACT OF A LOW-COST DIGITAL AND PRINT AWARENESS CAMPAIGN ON PATIENT BEHAVIOUR IN RELATION TO PERSONAL RISK OF OSTEOPOROSIS AND FRAGILITY FRACTURE}

H. Mace, C. Bovey, H. Arden, T. Jones. National Osteoporosis Society, Bath, UK

Background: The National Osteoporosis Society (NOS) 'Stop at One' campaign aims to encourage people who are over 50 and have broken a bone to find out, by taking an online quiz, if they are at risk of osteoporosis and to take action to reduce their risk of further fractures. A low-cost marketing intervention was trialled making printed campaign materials available direct to patients at the point of care (fracture clinic).

Objectives: The analysis sought to establish whether the marketing intervention increased take up of the online quiz, and to what extent taking the quiz influenced people's behaviour with regard to their bone health.

Methods: Between May and October 2017, the NOS placed Stop at One printed campaign materials encouraging people to take the online bone health quiz at 8 sites across the UK covering $13 \%$ (16/124) of UK postcode areas. 7 sites had no enhanced provision for secondary fracture prevention such as a fracture liaison service, 1 had a partial service. People who took the online quiz were sent a follow-up survey one month later.
Results: Up to 1st, January 20181909 people took the quiz:

$89 \%(1699)$ of these were over 50

$95 \%$ (1814) were female.

$21 \%$ (443) of these lived in postcode areas of the pilot sites.

1359 people were sent a follow up survey one month after taking the online quiz, and $10 \%(142)$ completed it. $27 \%{ }^{39}$ of these were individuals living in the postcode areas of the pilot sites.

When surveyed, of the 142 respondents:

$-50 \%(71)$ of respondents had broken a bone in the previous ten years.

$-73 \%(104)$ thought they were at risk of osteoporosis after taking the test.

$24 \%{ }^{34}$ had either booked or attended an appointment with their GP to discuss their possible risk of osteoporosis.

A further $10 \%{ }^{14}$ planned to book a GP appointment to discuss their risk. $31 \%{ }^{43}$ had made changes to their exercise habits.

- 33\% ${ }^{45}$ had made changes to their diet.

Conclusions: Digital activity (patients accessing website) and the electronic patient survey show meaningful changes in patient behaviour to reduce their fracture risk. Visibility of the awareness campaign at the point of care increased uptake of the quiz and subsequent survey.

Disclosure of Interest: None declared

DOI: 10.1136/annrheumdis-2018-eular.3729

\section{AB0990 THREE YEARS IMPROVEMENT IN OSTEOPOROSIS TREATMENT ADHERENCE FOLLOWING A THERAPEUTIC PATIENT EDUCATION (TPE) PROGRAM}

D. Poivret ${ }^{1}$, V. Noirez ${ }^{1}$, C. Goetz ${ }^{1}$, C. Wilcke ${ }^{2} .{ }^{1}$ CHR METZ THIONVILLE; ${ }^{2}$ URPS Grand Est, Metz, France

Background: The management of osteoporosis requires a restrictive drug treatment over several years and lifestyle changes: maintaining a suitable physical activity, a sufficient calcium intake and maintenance of ground balance. Unfortunately, treatment adherence at one year doesn't exceed $50 \%$. The education of the patient allows to take care of himself in his therapeutic approach but requires a relay to continue the follow-up over several years.

Objectives: To improve the follow-up of the patient, we created a cooperation between the doctor and the pharmacist.

Methods: We suggested to patients treated for osteoporosis by diphosphonate, denosumab or teriparatide to participate at two half-day TPE sessions, one year apart. It's taught that osteoporosis requires at least 5 years of treatment, and must be associated with the absorption of three daily dairy products, maintaining physical activity and preventing falls.

A follow-up notebook that contains 6 doctor and 6 pharmacist questionnaires is given to each patient participating in a TPE session. The patient sends his book to the doctor, and then to the pharmacist twice a year for three years. The pharmacist questionnaire explains the drug intake. The doctor questionnaire evokes lifestyle changes. This one is completed by the patient in the waiting room and is discussed with the doctor during the consultation. Each completed questionnaire provides a financial compensation to the health professional.

The program is funded by the Regional Health Agence of Lorraine and by the Regional Union of Health Professionals (doctors and pharmacists). Inclusions started in January 2013. We studied the results of the 3 year questionnaires for 94 patients included in 2013 and 2014.

Results: Among the 94 patients, only 49 continue their treatment at 3 years. Patients who stopped treatment, 4 died, 1 presented an atypical fracture of the femoral shaft, 6 stopped due to dental treatment, 4 had a contraindication to any anti-osteoporosis treatment, 1stopped treatment due to multiple sclerosis $11 \mathrm{left}$ the program, 2 had poorly tolerated the infusion and 1 discontinued treatment because of improved bone mineral density (BMD). Among the 49 patients who continue their treatment, 13 returned a doctor and pharmacist questionnaire at 3 years. There are 4 doctors and 2 pharmacists who refused to fill out the questionnaire, 20 patients didn't agree to respond to our request for news about their health condition and 4 patients lost to follow-up.

The study of 13 questionnaires received shows that at 3 years, 38\% of patients continue to consume 3 daily dairy products against $56 \%$ at 6 months. Nearly $70 \%$ of patients maintained their physical activity, $8 \%$ improved and $8 \%$ decreased it. It also shows that $46 \%$ of patients walk more than $30 \mathrm{~min}$ per day. The ground balance is satisfactory for $62 \%$ of patients compared to $71 \%$ at 6 months. Regarding compliance, at 6 months and 3 years, only $69 \%$ of patients never forget their treatment and $15 \%$ wanted to stop it. During the 3 years of follow-up, all patients were re-contacted at least once.

Conclusions: $52 \%$ of patients included continue the treatment at 3 years. $21 \%$ refuse to participate in the program. The doctor/pharmacist collaboration around the patient requires time and understanding. Patients recall and their participation in a research program improves their adherence to treatment and lifestyle changes.

Disclosure of Interest: None declared 


\section{AB0991 OSTEOPOROSIS AND FRACTURES IN PATIENTS WITH CIRRHOSIS. CAN FRAX BE USEFUL FOR SCREENING?}

E. Casado ${ }^{1}$, M. Arévalo ${ }^{1}$, J. Profitós ${ }^{2}$, A. Lira ${ }^{3}$, L. Del Río ${ }^{4}$, O. Valero ${ }^{5}$, M. Larrosa ${ }^{1}$, J. Sánchez-Delgado ${ }^{3}$, J. Gratacós ${ }^{1} .{ }^{1}$ Rheumatology, University Hospital Parc Taulí. I3PT. UAB. Sabadell, Sabadell; ${ }^{2}$ Gastroenterology and Hepatology, Consorci hospitalari de Terrassa, Terrassa; ${ }^{3}$ Gastroenterology and Hepatology, University Hospital Parc Taulí. I3PT. UAB. Sabadell, Sabadell; ${ }^{4}$ Radiolgy, CETIR Centre Mèdic, Barcelona; ${ }^{5}$ Statistics, Universitat Autònoma Barcelona (UAB), Bellaterra, Spain

Background: Osteoporotic fractures are a serious complication in patients with cirrhosis. In addition to the high morbidity and mortality of the patients who suffer them, fragility fractures represent a high cost for Healthcare systems.

However, there are very few studies that evaluate the prevalence of osteoporosis and fractures in patients with liver cirrhosis different than primary biliary cirrhosis (non-PBC cirrhosis). There are also no clinical guidelines with recommendations for osteoporosis screening in these patients.

Objectives: To assess the prevalence of osteoporosis and fragility fractures in patients with non-PBC cirrhosis in our environment, and the associated risk factors.

To analyse if the FRAX tool can be useful in the diagnostic screening of these patients.

Methods: From November 2015 to September 2017, outpatients older than 40 years diagnosed with non-PBC cirrhosis (any Child stage) were randomly included.

Demographic, clinical and analytical data (calcium, phosphorus, 25-hydroxyvitamin $D$ and PTH) were collected from all patients. A bone densitometry, GE, Lunar Prodigy (DXA) and vertebral fracture assessment (VFA) were also performed, for the diagnosis of osteoporosis (T-score $\leq-2.5$ ), and vertebral fracture. The 10 year absolute fracture risk was calculated using FRAX (https://www.sheffield. ac.uk/FRAX/tool.aspx? country=4).

A descriptive statistic of the main variables was carried out, with univariate and multivariate analysis to assess which predictive factors could be related to the presence of osteoporosis and/or fragility fractures.

Results: Ninety-two patients were included (71\% male and 29\% female). Age 63 \pm 11 years. The etiology of cirrhosis was: alcohol $(52 \%)$, hepatitis $C$ virus $(27 \%)$ and alcohol thepatitis C virus (9\%). Stage: Child A (80.4\%), B (17.4\%) and C (2.2\%). Mean 25 -hydroxyvitamin D was $18.5 \pm 9.8 \mathrm{ng} / \mathrm{ml}$ and PTH $51.8 \pm 23.0 \mathrm{pg} /$ $\mathrm{ml}$.

16 patients (17\%) had osteoporosis by DXA, 54 patients $(59 \%)$ osteopenia and 22 $(24 \%)$ had a normal bone mineral density (BMD). 8 patients $(9 \%)$ had suffered some fragility fracture (vertebral fracture in 6 cases).

The 10 year absolute risk for major fracture (vertebra, humerus, femur or radius) by FRAX without BMD was $5.7+4.5$; and with BMD $4.7 \pm 4.9$.

Age and female sex were associated with the presence of osteoporosis, and a $\mathrm{BMI}$ higher than 30 was found to be a protective factor. A BMD in the range of osteoporosis was the only factor associated with fracture.

FRAX for major fracture without BMD higher than $6.6 \%$ in this population had a high sensitivity (69\%) and specificity (85\%) for the diagnosis of osteoporosis, which implies a negative predictive value of $93 \%$. Using this FRAX cut-off for indicating DXA in cirrhotic patients could expect a saving of $76 \%$ of DXA scans.

Conclusions: The prevalence of osteoporosis and fractures in patients with nonPBC cirrhosis, even in mild stages, is higher than in the healthy population, being more frequent in women and older patients.

The FRAX tool can be useful in the selection of patients with cirrhosis to be assessed by a bone densitometry.

Disclosure of Interest: None declared

DOI: 10.1136/annrheumdis-2018-eular.4072

\section{AB0992 BONE REMODELLING BIOMARKERS IN HIV INFECTED PATIENTS}

F. López ${ }^{1}$, J. Loarce ${ }^{1}$, C. Sobrino ${ }^{1}$, J.L. Casado ${ }^{2}$, M. Vázquez ${ }^{1}{ }^{1}$ Rheumatology $^{2}$ Department, ${ }^{2}$ Infectious Diseases Department, Ramon y Cajal University Hospital, Madrid, Spain

Background: Bone metabolism is an equilibrium of resorption and growth, maintained by many regulating factors. Several molecules have been identified that estimate bone turnover, being P1NP and b-CTX the most commonly used. Many studies have shown a relationship between their levels and metabolic bone disease, and possibly with risk of fracture.

Human Immunodeficiency Virus (HIV) infected patients have lower bone mineral density (BMD), as documented on many studies. An increased incidence of fractures has been noted as well, probably due to predisposing factors related to HIV infection, apart from the traditional risk factors.

Objectives: To assess the relationship between mean values of bone biomarkers P1NP and b-CTX and incidence of vertebral fractures (VF) in a HIV infected population; and compare such values with those of a Spanish healthy population of reference.

Methods: We performed a cross-sectional study with HIV infected patients followed up in the Infectious Diseases Department of our centre from 2014 to 2016 P1NP and $b-C T X$ values were determined and lumbar and thoracic spine radiographs made to assess presence of VF (Genant grading scale). Other clinical and demographic data were collected retrospectively. P1NP and b-CTX values in the presence (VF group) and absence of fractures (non-FV group) were compared. Mean values were also compared with the Camargo cohort, comprised of 1080 healthy postmenopausal Spanish women, used as reference. Statistical analysis were made with STATA. All patients signed and informed consent, previously approved by the Hospital's Ethics Comittee.

Results: A total of 144 patients were included, 38 were women with a mean age of 56,4 years old ${ }^{45-77}$ and 106 men with mean age of 56,5 years old. ${ }^{45-86} 27$ of the patients had at least one VF. No statistically significant differences were found between P1NP mean levels in the FV and the non FV groups, with values of $45,30 \mathrm{ng} / \mathrm{ml} \quad( \pm 17,59 \mathrm{ng} / \mathrm{ml})$ and $49.48 \mathrm{ng} / \mathrm{ml} \quad( \pm 32,92 \mathrm{ng} / \mathrm{ml})$ respectively, $(\mathrm{p}=0.52)$. Mean levels of $\mathrm{b}-\mathrm{CTX}$ were $0,38 \mathrm{ng} / \mathrm{ml}( \pm 0,18 \mathrm{ng} / \mathrm{ml})$ in the VF group and $0,43 \mathrm{ng} / \mathrm{ml}( \pm 0,22 \mathrm{ng} / \mathrm{ml})$ in the non-FV group, again without significant differences $(p=0,35)$.

Mean general b-CTX values in our population were $0,41 \mathrm{ng} / \mathrm{ml}( \pm 0,21) ; 0,46 \mathrm{ng}$ $\mathrm{ml}( \pm 0,20)$ in women and $0,39 \mathrm{ng} / \mathrm{ml}( \pm 0,20)$ in men. Higher levels were found in HIV infected women than in the Camargo cohort $(0,38 \pm 0,19 \mathrm{ng} / \mathrm{ml})$, with statistical significance $(p=0.03)$. Mean general P1NP values were $48.34 \mathrm{ng} / \mathrm{ml}( \pm 29.47)$ $58.63 \mathrm{ng} / \mathrm{ml}( \pm 32.9)$ in women and $44.95 \mathrm{ng} / \mathrm{ml}( \pm 27.56)$ in men, with no statistically significant differences found when HIV infected women were compared with those of the Camargo cohort, $(47,7 \pm 19,9 \mathrm{ng} / \mathrm{ml})(\mathrm{p}=0.06)$, although a trend towards higher levels in HIV infected women was observed.

Conclusions: In the present study no correlation between P1NP and b-CTX levels in HIV infected patients and incidence of vertebral fracture was found.

P1NP and b-CTX mean values in HIV infected women in our study are higher than those of healthy postmenopausal Spanish women, which means a higher bone turnover in this population. More studies are needed to clarify the extent and clinical impact of this finding.

Disclosure of Interest: None declared

DOI: 10.1136/annrheumdis-2018-eular.5853

\section{AB0993 PAIN RELIEF MANAGEMENT OF ACUTE OSTEOPOROTIC VERTEBRAL FRACTURE IN A REAL LIFE STUDY}

F. Bertoldo ${ }^{1}$, G. Gandolini ${ }^{2}$, A. Venturin ${ }^{3}$, C. Cisari ${ }^{4}$, R. Lovato ${ }^{5}$, M. Longhi ${ }^{6}$, S. Farina ${ }^{7}$, E. Bertoldo ${ }^{8}$, R. Nuti ${ }^{9}$, on behalf of Servatius GISMO study group. ${ }^{1}$ Internal Medicine, University of Verona, Verona; ${ }^{2}$ rheumatology, IRCCS Don Gnocchi, Milan; ${ }^{3}$ Rheabiltation Unit, University of Padua, Padua; ${ }^{4}$ Orthopedic Unit, University of PO, Novara; ${ }^{5}$ Internal Medicine, Villa Berica Hospital, Vicenza; ${ }^{6}$ Orthopedic Unit, Galeazzi Institute, MIlan; ${ }^{7}$ Rheabiltation Unit, Crema Hospital, Crema; ${ }^{8}$ Rheumatology Unit, University of Verona, Verona; ${ }^{9}$ Internal Medicine, University of Siena, Siena, Italy

Background: Among all osteoporotic fractures the painful vertebral fractures (PVF) are the minority and their management is challenging for the clinician because the evidences about the best approach are conflicting and of low quality. Moreover there are no guidelines or consensus of experts. A real life picture of the management of the PVF is lacking in the literature.

Objectives: The primary end point of the study is to describe the pharmacological and or non-pharmacological management of PVF through the record of use of individual classes, associations and sequences of drugs or procedures. Secondary end point is the outcome of treatment in term of pain, disabilities and quality of life.

Methods: We present the iterim analysis results of a multicentric cross-sectional observational study. 400 interviews will be collected consecutively about pain, disability, pharmacological, spinal orthoses and orthopaedic surgery after the diag nosis of PFV in postmenopausal women treated by orthopaedics, endocrinologists, geriatricians, physiatrists, neurosurgeons and E.R. pysicians. Pain and disability were quantified by NRS scale and by QUALEFFO-41. Data collected from the first 100 patients have been analysed.

Results: One hundred postmenopausal women aged $73.1 \pm 7.49$ y.o (age of menopause $48.6 \pm 3.9$ years, L1-L4 T-score $-2.75 \pm 0.92$, Total hip T-score -2.35 \pm 0.98 ) with a new or first PFV were recorded. About $49 \%$ of them had a previous vertebral fracture and $22 \%$ a previous non-vertebral fragility fracture, $27 \%$ was not on osteoporosis treatment. The interviews were collected $135 \pm 114$ days from diagnosis of $P V, 174 \pm 141$ days from onset of pain. $92.3 \% \pm 12 \%$ of subjects were treated for pain relief for a mean time of $98 \pm 12$ days. Only $1.6 \%$ of cohort 
underwent to percutaneous vertebroplasty while $84.6 \% \pm 17.1 \%$ had spinal orthoses. Pharmacological treatment for pain was prescibed to $98.2+7.1 \%$ of subjects: acetaminophen (42\%), tapentadol (24\%), opioids (24\%), NSAID (6\%) and codeine with acetaminophen (4\%). In $95 \%$ of patients with spinal orthoses drugs for pain were assumed. In about $40 \%$ of cases NSAID was switched to acetaminophen, in $18 \%$ opioid and tapentadol switched to NSAID or acetaminophen. Only a few titration of opioids/tapentadol were reported. Not adequate pain relief (NRS scale 6.2 \pm 3.1 ; QUALEFFO-41 pain score $70 \pm 14.2$ ) and impairment quality of life (mean total QUALEFFO-41 score 65.1 \pm 20.1 ) were reported.

Conclusions: With the limits of the study design and low number of cases, preliminary data seem to confirm an inadequate pain relief in PVF. The emerging critical issues crossing all cathegories of physicians are the lag of diagnosis, the inappropiate use of acetaminophen, the missing tritation of opioids or tapentadol. A definition of optimal management of acute vertebral fracture is missing due to conflicting and scarce evidences in this field predisposing to chronic pain and disability.

Disclosure of Interest: None declared

DOI: 10.1136/annrheumdis-2018-eular.5427

\section{AB0994 IMPACT ON THE ADHERENCE AND PERSISTENCE OF DENOSUMAB VS WEEKLY BISPHOSPHONATE IN HEALTH-RELATED QUALITY OF LIFE IN POSTMENOPAUSAL OSTEOPOROSIS}

G. Carlino ${ }^{1}$, R. Cinzia ${ }^{1}$, R. Maggio ${ }^{2} .{ }^{1}$ Rheumatology, ASL LE, Casarano; ${ }^{2}$ Internal Medicine, "Veris Delli Ponti" Hospital, Scorrano, Italy

Background: Long term adherence and persistence in patients undergoing treatment for postmenopausal osteoporosis remains poor despite the proven efficacy of the therapy.

Objectives: In this study, we evaluated whether greater adherence and persistence in treatment can lead to an improvement in the quality of life.

Methods: A cohort of 268 patients, all women, in postmenopausal osteoporosis divided into two groups was evaluated: "DEN Group" (DEN) in treatment with denosumab ( $n=131)$ and "BIS Group" (BIS) in treatment with bisphosphonates $(n=137)$. Table 1 shows demographic and clinical data. Patients were followed for 3 years with baseline, 6 month, 18 month, and 36 month evaluation. The evaluation criteria were the persistence in therapy and the self-related treatment compliance, as well as the quality of life assessed with the 41-item Quality of Life questionnaire for osteoporosis (QUALEFFO-41) performed at baseline, at 18 and at 36 months.

Results: Table 2 shows the percentage of patients who abandoned treatment at different times with a statistical significance towards both 18 and 36 months. In the BIS the main reason for abandonment were the adverse events (gastrointestinal, dental interventions, etc.), in the DEN the abandonment was due to drop-out. [In BIS, the most frequent reason for non-compliance with therapy was oversight, and most patients who continued treatment always used the drugs regularly on the recommended days and dosages.] In the period of time considered the majority of patients both BIS and DEN said they were satisfied with the treatment and wanted to continue it. The QUALEFFO scores (Fig 1) of patients from the baseline visit were significantly improved in the 36 month visit (BIS $79.6 \pm 25.4$ vs 65.4 \pm 14.6 ; DEN $80.2 \pm 18.5$ vs $55.6^{*} \pm 16.4\left({ }^{*} \mathrm{p}<0.001\right)$; the difference was not significant between BIS and DEN groups at both baseline visit and 36 month visit, but in the DEN group there was significance between baseline and 36 month visit.

Abstract AB0994 - Table 1. Baseline characteristics (Mean \pm SD)

$\mathrm{OC}=$ Osteocalcin, $\mathrm{CTX}=\mathrm{C}$ terminal telopeptide, $\mathrm{PTH}=$ parathyroid hormone,

25OHD=25 hydroxyvitamin D;

Abstract AB0994 - Table 2. Percentage of patients who abandoned.

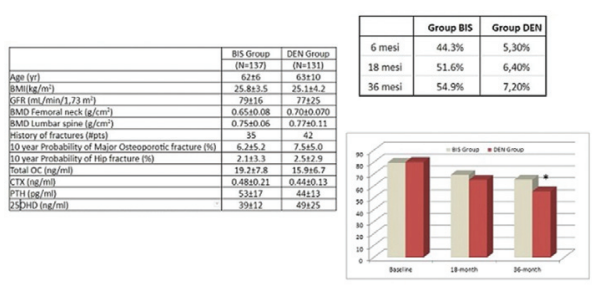

Abstract AB0994 - Figure 1. Qualeffo-41 Score $\left({ }^{*}\right.$ see text)
Conclusions: In conclusion, the observation, although numerically limited, notes that the use of denosumab in patients with postmenopausal osteoporosis leads to a greater persistence in treatment and a statistically significant adherence to therapy, which allows to obtain the maximum therapeutic effect of the therapy, also determining in 36 months of treatment an improvement in the quality of life, which is not achieved in subjects treated with bisphosphonates.

Disclosure of Interest: None declared

DOI: 10.1136/annrheumdis-2018-eular.3982

\section{AB0995 VERTEBRAL FRACTURES CASCADE: POTENTIAL ETIOLOGIES AND RISK FACTORS}

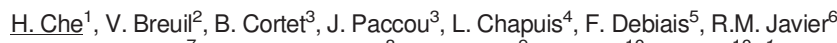
N. Mehsen Cetre ${ }^{7}$, S. Loiseau Peres ${ }^{8}$, T. Thomas ${ }^{9}$, C. Roux ${ }^{10}, \mathrm{~K}_{\text {B Briot }}{ }^{10} .{ }^{1} \mathrm{CHU}$ Lapeyronie, Montpellier, ${ }^{2} \mathrm{CHU}$ Nice, Nice; ${ }^{3} \mathrm{CHU}$ Lille, Lille; ${ }^{4} \mathrm{CH}$ du Vitre, Vitre;

${ }^{5} \mathrm{CHU}$ Poitiers, Poitiers; ${ }^{6} \mathrm{CHU}$ Hautepierre, Strasbourg; ${ }^{7} \mathrm{CHU}$ Bordeaux, Bordeaux; ${ }^{8} \mathrm{CHR}$ Orleans, Orleans: ${ }^{9} \mathrm{CHU}$ Saint Etienne, Saint Etienne; ${ }^{10} \mathrm{CHU}$ Cochin, Paris, France

Background: Vertebral fracture (VF) is the most common osteoporotic fracture, and a strong risk factor of subsequent vertebral fracture. Prospective studies have shown that a recent VF increases an imminent risk of a subsequent one, and attention has been paid recently to a possible cascade phenomenon i.e. the occurrence of multiples VFs in less than one year.

Objectives: This cascade could have severe consequences, and we prompted a study to identify potential causes of osteoporosis and risk factors.

Methods: Vertebral fractures cascade (VFC) observations were collected retrospectively between January 2016 and April 2017. VFC was defined as the occurrence of at least 3 vertebral fractures within one year. Patients with other etiologies than osteoporosis (i.e. malignant or traumatic VFs) were excluded. The cause of osteoporosis associated with VFC was the one retained by the physician at the time of diagnosis.

Results: Ninety-five observations of VFC ( $80 \%$ of women, mean age of 71 years) were collected in 10 centres ( 9 tertiary centres and 1 outpatient centre). The median number of incident VFs over 1 year was $4 .^{3-11}$ Forty-five patients $(45.9 \%)$ had a previous major fracture before the VFC and $65(70.7 \%)$ had densitometric osteoporosis (T-Score $\leq-2.5$ SD either at lumbar or femoral site). Eighteen (19\%) patients currently received oral glucocorticoids treatment at the time of VFC, with a mean daily dose of $20 \mathrm{mg}$. Thirty-three (35.1\%) patients received systemic glucocorticoids in the past. The main comorbidities were history of cancer $(n=19)$ and chronic inflammatory diseases $(n=21)$ including asthma $(n=7)$, chronic obstructive pulmonary disease $(n=7)$ and rheumatoid arthritis $(n=7)$.

A secondary osteoporosis associated with the cascade was diagnosed in 54 patients $(54.5 \%)$ with the following causes: glucocorticoid-induced osteoporosis $(n=22,23.7 \%$ ), benign hemopathies (mastocytosis, MGUS) $(n=7,7.1 \%)$, use of aromatase inhibitors $(n=3,3.1 \%)$, anorexia nervosa $(n=3,3.1 \%)$, alcoholism $(n=3$, $3.1 \%)$, pregnancy and lactation-associated osteoporosis $(n=2,2.1 \%)$, primary hyperparathyroidism $(n=2,2.1 \%)$ and hypercorticism $(n=1,1.1 \%)$. In addition, 11 cases $(11.3 \%$ ) were reported following a vertebroplasty procedure.

Primary either postmenopausal or idiopathic osteoporosis was diagnosed in 48 patients $(51.6 \%)$. A total of $29(29.6 \%)$ patients previously received an anti-osteoporotic treatment. In six patients $(6.3 \%)$, VFC occurred early (in the year) following discontinuation of an anti-osteoporotic treatment: 5 after denosumab and one 12 months after an infusion of zoledronic acid.

Conclusions: The results of this retrospective study show that almost half of VFC occurred in patients with secondary osteoporosis. While they suggest that a careful management has to be given to these patients in order to prevent VFC in these circumstances, prospective studies are needed to further explore the determinants of such a severe complication of osteoporosis.

Disclosure of Interest: None declared

DOI: 10.1136/annrheumdis-2018-eular.2543

\section{AB0996 1 BONE MINERAL DENSITY AT DIFFERENT SITES AS A PREDICTOR OF RIB FRACTURES: A CASE-CONTROL STUDY}

H.L. Wu, M. Bukhari. Rheumatology, University Hospitals of Morecambe Bay NHS Foundation Trust, Lancaster, UK

Background: Rib fractures commonly occur as a result of direct trauma, though pathological causes have also been identified. Literature on the specific risk factors of rib fractures is scarce. There was an American prospective cohort study 
which highlighted the relationship between risk factors of osteoporosis and rib fractures in older men aged 65 or above. ${ }^{1}$ It was observed that the incidence of rib fractures was $3.5 / 1000$ years. Only $3 \%$ of rib fractures occurred with absence of trauma. Bone mineral density (BMD) is an important measure for predicting various bone fractures. ${ }^{2}$ However, prediction of rib fractures using BMD measurement in different body sites is not reported.

Objectives: To determine if reduction in femoral neck and lumbar spine BMD are predictive of rib fractures.

Methods: Patients referred from primary care to a DEXA scanner in the north west of England between January 2006 and December 2016 were used in this study. Patients with a history of rib fractures at first scan were matched with controls who did not have any indication for scanning. Cases and controls were matched for age and gender. Differences in BMD at L1-L4 spine and the femoral neck were analysed using two-sample t test. Logistic regression models were fitted to analyse the association between lumbar spine and femoral neck BMD and rib fracture occurrence. The fit of each model was compared using receiver operating characteristic (ROC) curves.

Results: A total of 1554 patients were included in the study ( 777 cases of rib fractures and 777 controls). Mean age for both cases and controls were 62.5 years (SD 12.0). 605 patients (77.9\%) were female in both the case cohort and the controls. The mean T score in the lumbar spine is 1.00 in cases versus 1.10 in controls (diff $0.10095 \% \mathrm{Cl} 0.0488,0.142 \mathrm{p}<0.001$ ). The mean T score in the femoral neck is 0.812 in cases versus 0.935 in controls (diff $0.12395 \% \mathrm{Cl} 0.108$, $0.137 \mathrm{p}<0.001)$. The odds of lumbar spine BMD and femoral neck BMD were 0.111 (95\% Cl 0.0640, 0.194, $\mathrm{p}<0.001)$ and 0.00209 (95\% Cl 0.000903, 0.00485, $\mathrm{p}<0.001)$ respectively. The areas under ROC curve (AUC) for lumbar spine BMD and femoral neck BMD were 0.623 and 0.733 .

Conclusions: This study demonstrated that reduction in BMD at the lumbar spine and femoral neck positively correlated to the risk of rib fractures. Reduction in femoral neck BMD is a stronger predictor of the two. Prediction of rib fractures could be affected by other factors influencing lumbar spine and femoral neck BMD. Further work in different demographic groups should be done for comparison and analysis.

\section{REFERENCES:}

[1] Barrett-Connor, et al. Epidemiology of rib fractures in older men: Osteoporotic Fractures in Men (MrOS) prospective cohort study. BMJ 2010; 340: c1069

[2] Dobrzynska, et al. Predictors of fragility humeral fractures: An Observational Study. Ann Rheum Dis 2015; 74(Suppl2):763

Disclosure of Interest: None declared

DOI: 10.1136/annrheumdis-2018-eular.1360

\section{AB0997 CLINICAL CHARACTERISTICS OF NON-RESPONDER TO DENOSUMAB TREATMENT FOR POSTMENOPAUSAL OSTEOPOROSIS IN JAPANESE WOMAN}

I. Yoshii. Orthopaedics, YOSHII HOSPITAL, Shimanto City, Japan

Background: Denosumab (d-mab), an anti-receptor activator of nuclear factor kappa-B ligand (RANKL) monoclonal antibody, is now widely used in postmenopausal osteoporosis (OP) treatment. It can attain reliable increase of bone mineral density (BMD) despite any OP drugs previous used, and that can be predicted in a long period. However, sometimes we observe BMP decrease. Factor that suppresses BMD gain is still unclear.

Objectives: This study investigates non-responder to d-mab, and attempted to evaluate factors that concerns non-responder in clinical practice retrospectively. Methods: 212 postmenopausal OP patients have been treated with d-mab continuously for more than two years under vitamin $D$ supplement and calcium since June 2013. These patients were enrolled, and their clinical background (CBs) such as age at start of d-mab, past history of bone fragility fracture, alcohol habitat, current smoking, being treated for rheumatoid arthritis (RA), glucocorticoid steroid thrown, OP drug naïve, length of administration (LA), concurrence of lifestyle disease (LSD) such as diabetes mellitus, hypertension, and chronic obstructive lung disease, and number of comorbidity (N.Com), were investigated (table 1a). BMD of lumbar spine (LS), femoral neck (FN), greater trochanter (GT), and whole femur $(\mathrm{WF})$ at the start and every six months thereafter were measured. Tartrate-resistant Acid Phosphatase 5b (TRACP5b) and total dose of type-one pro-collagen - $\mathrm{N}$ pro-peptide (P1NP) at the same time of BMD measurement, and reduction ratio $(R R)$ of them compared to the first shot were calculated. Patients were divided into two groups whether BMD increased. BMD in each part at final measurement relative to that at start was compared statistically with paired T-test. CBs were statistically evaluated between the two BMD groups (Res and n-Res; Responder and non-Responder) with Mann-Whitney U-test (MWT), and then correlation between the BMD groups and factors of $\mathrm{CB}$ that had demonstrated significantly difference, and TRACP5b and P1NP was also evaluated statistically with Binary Logistic Analysis (BLR). Statistical significance was set less than $5 \%$.

Results: For all patients, BMD in LS, WF, and GT demonstrated significant increase at final measurement than at start $(\mathrm{p}<0.001)$, while FN demonstrated no significant increase. However, n-Res counted 30, 89, 46, and 44 for LS, FN, WF, and GT, respectively. LA demonstrated negative significant correlation for being $\mathrm{n}$-Res in all part, while LSD, N.Com, TRACP5b at start, and RR of TRACP5b at second shot and last shot, demonstrated significant positive correlation in LS with MWT. However, with BLR, only RR of TRACP $5 b$ at second shot demonstrated significant negative correlation with n-Res in LS (OR:0.0702, 95\% Cl:0.00510.9655 ) with 0.39 cut-off index (COI), while no other factors but LA demonstrated significant negative correlation with n-Res in WF (OR:0.9669, 95\% Cl:0.94330.9911 ) and GT (OR:0.9632, 95\% Cl:0.9391-0.9878) with 26 and 24 months COI, respectively (table $1 \mathrm{~b}$ ).

Table 1a Patient's background. Age at Start, Length Administered, Number of Chronic Comorbidities, and Bone Mineral Density, show average value and standard deviation. The other parameters show numbers.\%YAM:\% of young adult mean value. LS: lumbar spine, FN: femoral neck, WF: whole femur, GT: greater trochanter, SERM: selective oestrogen receptor modulator

Table $1 b$ Parameters that demonstrated significant difference between responder and non-responder to denosumab, and their $p$-values, and results of these param eters with binary logistic regression analysis. LS: lumbar spine, FN: femoral neck WF: whole femur, GT: greater trochanter, LSD: concurrence of lifestyle disease LA: length of administration, N.Com: number of concurrent comorbidities, TRACP5b at start; tartrate-resistant acid phosphatase 5b (TRACP5b) at start of administration, rTRACP5b@1stP: reduction ratio of TRACP5b of second shot compared to the first, rTRACP5b@LT: reduction ratio to TRACP5b of last shot compared to the first, Drug Naive: initial drug as osteoporosis treatment, PINP total dose of type-one pro-collagen $-\mathrm{N}$ - pro-peptide.



Conclusions: These results suggest that non-responder to d-mab exists. Length of administration works for BMD to increase in femur, and concurrence of LSD, and N.Com are suggested to be risk factors in LS. Less reduction ratio of TRACP $5 \mathrm{~b}$ at second shot is referred for prediction of non-responder. However, there is no parameter that predicts non-responder before administration.

Disclosure of Interest: None declared

DOI: 10.1136/annrheumdis-2018-eular.1263

\section{AB0998 OSTEOPOROSIS PREVALENCE IN POST-MENOPAUSAL PATIENTS TREATED WITH AROMATASE INHIBITORS USING BONE MINERAL DENSITY VALUES FROM A SPANISH POPULATION}

J. Loarce-Martos ${ }^{1}$, W.A. Sifuentes-Giraldo ${ }^{1}$, L.V. Maldonado-Romero ${ }^{2}$, M. AhijónLana $^{1}$, C. Velázquez-Arce ${ }^{1}$, N. Martínez-Jañez ${ }^{3}$, M. Vázquez-Díaz ${ }^{1}$.

${ }^{1}$ Rheumatology, Ramon y Cajal Universitary Hospital, Madrid, Spain;

${ }^{2}$ Rheumatology, Hospital Teodoro Maldonado Carbo, Guayaquil, Ecuador,

${ }^{3}$ Oncology, Ramon y Cajal Universitary Hospital, Madrid, Spain

Background: The majority of cases of breast cancer $(\mathrm{BC})$ are hormone receptor positive and are sensitive to antiestrogen therapy. For postmenopausal women, adjuvant hormonal therapy with an aromatase inhibitor $(\mathrm{Al})$ is the standard of care, and is associated with greater prevalence of osteoporosis (OP) ${ }^{1}$. Definition of osteoporosis is made depending on the T-Score value, which is calculated according to the bone mass peak obtained from a reference population; in our country, BMD reference values in clinical practice are obtained from the National Health and Nutrition Examination Survey cohort (NHANES III) for femoral neck (FN) and those proportionated by the commercial brand Hologic for lumbar spine (LS), which may not be representative of our population and could be distorting the assessment of OP in our patients. 